\title{
MEROMORPHIC FUNCTION OF INFINITE ORDER WITH MAXIMUM DEFICIENCY SUM*
}

\author{
WeILING Xiong
}

\begin{abstract}
In this paper we prove the following theorem: Let $f(z)$ be a meromorphic function of infinite order. If $\sum_{a \neq \infty} \delta(a, f)+\delta(\infty, f)=2$, then for each positive integer $k$, we have $K\left(f^{(k)}\right)=2 k(1-\delta(\infty, f)) /(1+k-k \delta(\infty, f))$, where $K\left(f^{(k)}\right)=$ $\lim _{r \rightarrow \infty}\left(N\left(r, 1 / f^{(k)}\right)+N\left(r, f^{(k)}\right)\right) / T\left(r, f^{(k)}\right)$ exists. This result improves the results by S. K. Singh and V. N. Kulkarni [1] and Mingliang Fang [2].
\end{abstract}

\section{Introduction and results}

In this paper, we assume that $f(z)$ is a nonconstant meromorphic function in the complex plane $C$. We shall use the standard notations of the Nevanlinna theory of meromorphic functions (see [3]).

$$
T(r, f), m(r, f), N(r, f), \bar{N}(r, f), \delta(a, f), S(r, f) \text { and so on. }
$$

We shall also use the following notations (see [4]):

$$
\begin{array}{ll}
T_{0}(r, f)=\int_{1}^{r} \frac{T(t, f)}{t} d t, & N_{0}(r, f)=\int_{1}^{r} \frac{N(t, f)}{t} d t, \\
m_{0}(r, f)=\int_{1}^{r} \frac{m(t, f)}{t} d t, & S_{0}(r, f)=\int_{1}^{r} \frac{S(t, f)}{t} d t .
\end{array}
$$

Similarly, we use the notations $m_{0}(r, a, f)$ and $N_{0}(r, a, f)$. Set

$$
\begin{gathered}
\delta_{0}(a, f)=1-\limsup _{r \rightarrow \infty} \frac{N_{0}(r, a, f)}{T_{0}(r, a, f)}, \\
K\left(f^{(k)}\right)=\limsup _{r \rightarrow \infty} \frac{N\left(r, 1 / f^{(k)}\right)+N\left(r, f^{(k)}\right)}{T\left(r, f^{(k)}\right)}, \quad(k=0,1,2, \ldots) .
\end{gathered}
$$

In 1973, S. K. Singh and V. N. Kulkarni [1] proved the following result:

AMS Classification: 30D35.

Keywords: meromorphic function, infinite order, maximum deficiency sum.

* Supported by NSF of Guangxi China (0339018).

Received May 22, 2003; revised November 17, 2003. 
THEOREM 1.1. Suppose that $f$ is a transcendental meromorphic function of finite order satisfying

$$
\sum_{a \neq \infty} \delta(a, f)+\delta(\infty, f)=2
$$

Then

$$
\frac{1-\delta(\infty, f)}{2-\delta(\infty, f)} \leq K\left(f^{\prime}\right) \leq \frac{2(1-\delta(\infty, f))}{2-\delta(\infty, f)}
$$

In 2000, Mingliang Fang [2] proved the following result:

THEOREM 1.2. Suppose that $f$ is a transcendental meromorphic function of finite order satisfying

$$
\sum_{a \neq \infty} \delta(a, f)+\delta(\infty, f)=2
$$

Then

$$
K\left(f^{(k)}\right)=\frac{2 k(1-\delta(\infty, f))}{1+k-k \delta(\infty, f)} .
$$

In this paper, we shall prove the following theorem:

THEOREM 1.3. Suppose that $f$ is a meromorphic function of infinite order. If

$$
\sum_{a \neq \infty} \delta(a, f)+\delta(\infty, f)=2
$$

then for each positive integer $k$,

$$
K\left(f^{(k)}\right)=\frac{2 k(1-\delta(\infty, f))}{1+k-k \delta(\infty, f)} .
$$

\section{Lemmas}

Lemma 1 ([4]). Suppose that $f$ is a meromorphic function of infinite order. Then for each complex number $a$,

$$
0 \leq \delta(a, f) \leq \delta_{0}(a, f) \leq 1, \quad \sum_{a \neq \infty} \delta_{0}(a, f)+\delta_{0}(\infty, f) \leq 2 .
$$

Lemma 2 ([5]). Suppose that $f$ is a meromorphic function of infinite order satisfying $\sum_{a \neq \infty} \delta(a, f)+\delta(\infty, f)=2$. Then for each $k \in N$, 


$$
T_{0}\left(r, f^{(k)}\right)=\left((k+1)-k \delta_{0}(\infty, f)+o(1)\right) T_{0}(r, f),
$$

as $r \rightarrow \infty$ through all values.

Using Lemma 2, we can prove

Lemma 3 ([5]). Suppose that $f$ is a meromorphic function of infinite order satisfying $\sum_{a \neq \infty} \delta(a, f)+\delta(\infty, f)=2$. Then for each $k \in N$,

$$
T\left(r, f^{(k)}\right)=((k+1)-k \delta(\infty, f)+o(1)) T(r, f),
$$

as $r \rightarrow \infty$ through all values.

Proof. Because the paper [5] is written in Chinese, we give here a sketch of the proof of Lemma 3. Set $b=(k+1)-k \delta(\infty, f), F_{1}(x)=T_{0}\left(e^{x}, f^{(k)}\right)$ and $G_{1}=b \cdot T_{0}\left(e^{x}, f\right)$. Then by Lemma 2 and by a similar method to the part (II) of the proof of Theorem 1.3 below, we see that $F_{1}(x)$ and $G_{1}(x)$ satisfy the conditions of Lemma 9. Thus we have

$$
\lim _{x \rightarrow \infty} \frac{F_{1}^{\prime}(x)}{G_{1}^{\prime}(x)}=1 .
$$

Hence we obtain the conclusion of Lemma 3.

Lemma 4 ([6]). Suppose that $f$ is a transcendental meromorphic function, and $a_{i}(i=1,2, \ldots, p)$ be $p$ distinct complex numbers. Then for each $k \in N$,

$$
\sum_{i=1}^{p} m\left(r, a_{i}, f\right) \leq m\left(r, \frac{1}{f^{(k)}}\right)+S(r, f) .
$$

Lemma 5 ([6]). Suppose that $f$ is a transcendental meromorphic function of infinite order, $S(r, f)$ be any quantity satisfying

$$
S(r, f) \leq A \log ^{+} T(R, f)+B \log ^{+} \frac{1}{R-r}+C \log ^{+} R+D,
$$

where $0<r<R$, and $A, B, C, D$ are positive constants. Then $\lim _{r \rightarrow \infty} S_{0}(r, f) /$ $T_{0}(r, f)=0$.

Lemma 6 ([7]). Suppose that $f$ is a transcendental meromorphic function. Then for each positive number $\varepsilon_{0}$ and for each $k \in N$,

$$
\bar{N}(r, f)<\frac{1}{k} N\left(r, \frac{1}{f^{(k)}}\right)+\frac{1}{k} N(r, f)+\varepsilon_{0} T(r, f)+S(r, f) .
$$

Lemma 7. Suppose that $f$ is a meromorphic function of infinite order. If $\sum_{a \neq \infty} \delta_{0}(a, f)+\delta_{0}(\infty, f)=2$, then for each positive integer $k$, 


$$
\lim _{r \rightarrow \infty} \frac{m_{0}\left(r, 1 / f^{(k)}\right)}{T_{0}(r, f)}=2-\delta_{0}(\infty, f) \quad \text { and } \quad \lim _{r \rightarrow \infty} \frac{N_{0}(r, f)}{T_{0}(r, f)}=\lim _{r \rightarrow \infty} \frac{\bar{N}_{0}(r, f)}{T_{0}(r, f)} .
$$

Proof. Since $\sum_{a \neq \infty} \delta_{0}(a, f)+\delta_{0}(\infty, f)=2$, then for any positive number $\varepsilon_{0}$, there exist distinct complex numbers $a_{i}(i=1,2, \ldots, p)$ such that

$$
\sum_{i=1}^{p} \delta_{0}\left(a_{i}, f\right)+\delta_{0}(\infty, f) \geq 2-\varepsilon_{0} .
$$

By Lemma 4, we have

$$
\sum_{i=1}^{p} m\left(r, a_{i}, f\right) \leq m\left(r, \frac{1}{f^{(k)}}\right)+S(r, f) .
$$

From (1), (2) and Lemma 5, we have

$$
\liminf _{r \rightarrow \infty} \frac{m_{0}\left(r, 1 / f^{(k)}\right)}{T_{0}(r, f)} \geq \sum_{i=1}^{p} \delta_{0}\left(a_{i}, f\right) \geq 2-\delta_{0}(\infty, f)-\varepsilon_{0} .
$$

Taking $\varepsilon_{0} \rightarrow 0$, we deduce

$$
\liminf _{r \rightarrow \infty} \frac{m_{0}\left(r, 1 / f^{(k)}\right)}{T_{0}(r, f)} \geq 2-\delta_{0}(\infty, f) .
$$

On the other hand, by Lemma 6, we have

$$
\begin{aligned}
m\left(r, \frac{1}{f^{(k)}}\right) & \leq T\left(r, f^{(k)}\right)-N\left(r, \frac{1}{f^{(k)}}\right)+S(r, f) \\
& \leq T(r, f)+k \bar{N}(r, f)-N\left(r, \frac{1}{f^{(k)}}\right)+S(r, f) \\
& \leq T(r, f)+N(r, f)+k \varepsilon_{0} T(r, f)+S(r, f) .
\end{aligned}
$$

Thus we have

$$
m_{0}\left(r, \frac{1}{f^{(k)}}\right) \leq T_{0}(r, f)+N_{0}(r, f)+k \varepsilon_{0} T_{0}(r, f)+S_{0}(r, f) .
$$

Hence we obtain

$$
\limsup _{r \rightarrow \infty} \frac{m_{0}\left(r, 1 / f^{(k)}\right)}{T_{0}(r, f)} \leq 2-\delta_{0}(\infty, f)+k \varepsilon_{0} .
$$

Taking $\varepsilon_{0} \rightarrow 0$, we deduce

$$
\limsup _{r \rightarrow \infty} \frac{m_{0}\left(r, 1 / f^{(k)}\right)}{T_{0}(r, f)} \leq 2-\delta_{0}(\infty, f) .
$$


From (3) and (5), we have

$$
\lim _{r \rightarrow \infty} \frac{m_{0}\left(r, 1 / f^{(k)}\right)}{T_{0}(r, f)}=2-\delta_{0}(\infty, f) .
$$

Choosing $k=1$, from (2) and (4) we have

$$
\sum_{i=1}^{p} \delta_{0}\left(a_{i}, f\right) \leq 1+\liminf _{r \rightarrow \infty} \frac{\bar{N}_{0}(r, f)}{T_{0}(r, f)} \leq 1+\limsup _{r \rightarrow \infty} \frac{N_{0}(r, f)}{T_{0}(r, f)}=2-\delta_{0}(\infty, f) .
$$

From (1), we have

$$
2-\delta_{0}(\infty, f)-\varepsilon_{0} \leq \sum_{i=1}^{p} \delta_{0}\left(a_{i}, f\right)
$$

Taking $\varepsilon_{0} \rightarrow 0$, we obtain

$$
\liminf _{r \rightarrow \infty} \frac{\bar{N}_{0}(r, f)}{T_{0}(r, f)}=\limsup _{r \rightarrow \infty} \frac{N_{0}(r, f)}{T_{0}(r, f)},
$$

hence

$$
\lim _{r \rightarrow \infty} \frac{\bar{N}_{0}(r, f)}{T_{0}(r, f)}=\lim _{r \rightarrow \infty} \frac{N_{0}(r, f)}{T_{0}(r, f)} .
$$

This completes the proof of Lemma 7.

Lemma $8([8])$. Suppose that $f(r)$ is a nonnegative and increasing function with $\lim _{r \rightarrow \infty} f(r) / r^{\alpha}=\infty$ for each positive number $\alpha$. We set $F(x)=\int_{0}^{x} f(r) d r$. Then

$$
\lim _{x \rightarrow \infty} \frac{F(x)}{f^{2}(x) / f^{\prime}(x)}=1
$$

Lemma $9([9])$. Let $f(x)$ and $g(x)$ satisfy the following four conditions for $x \geq 0$ :

(i) $f^{\prime}(x)$ and $g^{\prime}(x)$ are two continuous functions

(ii) $f(x)$ is an increasing convex function

(iii) $1 / g(x)$ is a convex function

(iv) $\lim _{x \rightarrow \infty} f^{\prime}(x) / g^{\prime}(x)=1$.

Then $\lim _{x \rightarrow \infty} f^{\prime}(x) / g^{\prime}(x)=1$.

\section{Proof of Theorem 1.3}

Since $\sum_{a \neq \infty} \delta(a, f)+\delta(\infty, f)=2$, by Lemma 1 and Lemma 3, we have $\delta(\infty, f)=\delta_{0}(\infty, f)$ and

$$
T\left(r, f^{(k)}\right)=((k+1)-k \delta(\infty, f)+o(1)) T(r, f),
$$

as $r \rightarrow \infty$ through all values. 
From (6), Lemma 3 and Lemma 7, we have

$$
\begin{aligned}
& \lim _{r \rightarrow \infty} \frac{m_{0}\left(r, 1 / f^{(k)}\right)}{T_{0}(r, f)}=2-\delta_{0}(\infty, f), \\
& \lim _{r \rightarrow \infty} \frac{N_{0}\left(r, 1 / f^{(k)}\right)}{T_{0}\left(r, f^{(k)}\right)}=1-\lim _{r \rightarrow \infty} \frac{m_{0}\left(r, 1 / f^{(k)}\right)}{T_{0}(r, f)} \lim _{r \rightarrow \infty} \frac{T_{0}(r, f)}{T_{0}\left(r, f^{(k)}\right)}=A_{0}, \\
& \lim _{r \rightarrow \infty} \frac{N_{0}\left(r, f^{(k)}\right)}{T_{0}\left(r, f^{(k)}\right)}=\lim _{r \rightarrow \infty} \frac{N_{0}(r, f)+k \bar{N}_{0}(r, f)}{T_{0}\left(r, f^{(k)}\right)} \\
&=\lim _{r \rightarrow \infty} \frac{(k+1) N_{0}(r, f)}{T_{0}(r, f)} \lim _{r \rightarrow \infty} \frac{T_{0}(r, f)}{T_{0}\left(r, f^{(k)}\right)}=B_{0},
\end{aligned}
$$

where $\quad A_{0}=(k-1)(1-\delta(\infty, f)) /(1+k-k \delta(\infty, f)) \quad$ and $\quad B_{0}=(k+1)$. $(1-\delta(\infty, f)) /(1+k-k \delta(\infty, f))$.

(I) We shall first prove that either for any positive number $\beta$

$$
\lim _{r \rightarrow \infty} \frac{N\left(r, 1 / f^{(k)}\right)}{r^{\beta}}=\infty \text { and } \lim _{r \rightarrow \infty} \frac{N\left(r, f^{(k)}\right)}{r^{\beta}}=\infty,
$$

or the conclusion of Theorem 1.3 holds. Since $\sum_{a \neq \infty} \delta(a, f)+\delta(\infty, f)=2$, $f(z)$ is of regular growth [6]. Note that $f(z)$ is a meromorphic function of infinite order and regular growth. Then for any positive number $\beta$, we have $\lim _{r \rightarrow \infty} T\left(r, f^{(k)}\right) / r^{\beta}=\infty$. Thus we have

$$
\lim _{r \rightarrow \infty} \frac{T\left(r, 1 / f^{(k)}\right)}{r^{\beta}}=\infty .
$$

(i) If

$$
\liminf _{r \rightarrow \infty} \frac{N\left(r, 1 / f^{(k)}\right)}{T\left(r, f^{(k)}\right)}=B \neq 0,
$$

then

$$
\liminf _{r \rightarrow \infty} \frac{N\left(r, 1 / f^{(k)}\right)}{r^{\beta}} \geq \liminf _{r \rightarrow \infty} \frac{N\left(r, 1 / f^{(k)}\right)}{T\left(r, f^{(k)}\right)} \liminf _{r \rightarrow \infty} \frac{T\left(r, f^{(k)}\right)}{r^{\beta}}=\infty .
$$

Thus we have $\lim _{r \rightarrow \infty} N\left(r, 1 / f^{(k)}\right) / r^{\beta}=\infty$.

(ii) If

then

$$
\limsup _{r \rightarrow \infty} \frac{N\left(r, 1 / f^{(k)}\right)}{T\left(r, f^{(k)}\right)}=0
$$

$$
\lim _{r \rightarrow \infty} \frac{N\left(r, 1 / f^{(k)}\right)}{T\left(r, f^{(k)}\right)}=0 \quad \text { and } \quad \lim _{r \rightarrow \infty} \frac{m\left(r, 1 / f^{(k)}\right)}{T\left(r, f^{(k)}\right)}=1 .
$$

Thus we have 


$$
\lim _{r \rightarrow \infty} \frac{m_{0}\left(r, 1 / f^{(k)}\right)}{T_{0}\left(r, f^{(k)}\right)}=1 .
$$

From (6) and (7), we obtain $\delta(\infty, f)=1$. Therefore the conclusion of Theorem 1.3 holds.

(iii) Suppose that

$$
\limsup _{r \rightarrow \infty} \frac{N\left(r, 1 / f^{(k)}\right)}{T\left(r, f^{(k)}\right)}=B \neq 0 \quad \text { and } \quad \liminf _{r \rightarrow \infty} \frac{N\left(r, 1 / f^{(k)}\right)}{T\left(r, f^{(k)}\right)}=0 .
$$

Then we have

$$
\limsup _{r \rightarrow \infty} \frac{m\left(r, 1 / f^{(k)}\right)}{T\left(r, f^{(k)}\right)}=1 .
$$

Hence there exists an increasing sequence $r_{n} \rightarrow \infty$ such that $\lim _{n \rightarrow \infty} m\left(r_{n}, 1 / f^{(k)}\right) /$ $T\left(r_{n}, f^{(k)}\right)=1$. By (6), we have

$$
\lim _{n \rightarrow \infty} \frac{m\left(r_{n}, 1 / f^{(k)}\right)}{T\left(r_{n}, f\right)}=1+k-k \delta(\infty, f) .
$$

Hence by (7) and (11), we have

$$
\begin{aligned}
2-\delta(\infty, f) & =\lim _{r \rightarrow \infty} \frac{m_{0}\left(r, 1 / f^{k}\right)}{T_{0}(r, f)}=\lim _{n \rightarrow \infty} \frac{m_{0}\left(r_{n}, 1 / f^{k}\right)}{T_{0}\left(r_{n}, f\right)} \\
& =\lim _{n \rightarrow \infty} \frac{m\left(r_{n}, 1 / f^{k}\right)}{T\left(r_{n}, f\right)}=1+k-k \delta(\infty, f) .
\end{aligned}
$$

This yields $\delta(\infty, f)=1$. Hence $\delta_{0}(\infty, f)=1$ by Lemma 1 . Thus we have

$$
\begin{aligned}
\delta_{0}\left(0, f^{(k)}\right) & =\liminf _{r \rightarrow \infty} \frac{m_{0}\left(r, 1 / f^{(k)}\right)}{T_{0}\left(r, f^{(k)}\right)} \\
& =\liminf _{r \rightarrow \infty} \frac{m_{0}\left(r, 1 / f^{(k)}\right)}{T_{0}(r, f)} \lim _{r \rightarrow \infty} \frac{T_{0}(r, f)}{T_{0}\left(r, f^{(k)}\right)}=\frac{2-\delta_{0}(\infty, f)}{(k+1)-k \delta(\infty, f)}=1
\end{aligned}
$$

by $\delta(\infty, f)=\delta_{0}(\infty, f)=1$. Then

$$
1=\delta_{0}\left(0, f^{(k)}\right)=\liminf _{r \rightarrow \infty} \frac{m_{0}\left(r, 1 / f^{(k)}\right)}{T_{0}\left(r, f^{(k)}\right)} \leq \limsup _{r \rightarrow \infty} \frac{m_{0}\left(r, 1 / f^{(k)}\right)}{T_{0}\left(r, f^{(k)}\right)} \leq 1 .
$$

Thus $\lim _{r \rightarrow \infty} m_{0}\left(r, 1 / f^{(k)}\right) / T_{0}\left(r, f^{(k)}\right)$ exists. Therefore by using l'Hospital's rule, we obtain

$$
\delta_{0}\left(0, f^{(k)}\right)=\lim _{r \rightarrow \infty} \frac{m_{0}\left(r, 1 / f^{(k)}\right)}{T_{0}\left(r, f^{(k)}\right)}=\lim _{r \rightarrow \infty} \frac{m_{0}^{\prime}\left(r, 1 / f^{(k)}\right)}{T_{0}^{\prime}\left(r, f^{(k)}\right)}=\lim _{r \rightarrow \infty} \frac{m\left(r, 1 / f^{(k)}\right)}{T\left(r, f^{(k)}\right)}=\delta\left(0, f^{(k)}\right),
$$

that is, $\delta\left(0, f^{(k)}\right)=1$. Hence $\lim _{r \rightarrow \infty} N\left(r, 1 / f^{(k)}\right) / T\left(r, f^{(k)}\right)=0$. This is a contradiction. Therefore we deduce that $\lim _{r \rightarrow \infty} N\left(r, 1 / f^{(k)}\right) / r^{\beta}=\infty$ or the conclusion of Theorem 1.3 holds in this case. 
Similarly, we have $\lim _{r \rightarrow \infty} N\left(r, f^{(k)}\right) / r^{\beta}=\infty$ or the conclusion of Theorem 1.3 holds.

(II) Let $F(x)=N_{0}\left(e^{x}, 1 / f^{(k)}\right)$ and $G(x)=A_{0} T_{0}\left(e^{x}, f^{(k)}\right)$. Then we have

$$
\begin{gathered}
F(x)=\int_{1}^{e^{x}} \frac{N\left(t, 1 / f^{(k)}\right)}{t} d t=\int_{0}^{x} N\left(e^{r}, \frac{1}{f^{(k)}}\right) d r, \\
G(x)=A_{0} \int_{1}^{e^{x}} \frac{T\left(t, f^{(k)}\right)}{t} d t=A_{0} \int_{0}^{x} T\left(e^{r}, f^{(k)}\right) d r .
\end{gathered}
$$

Since $N\left(e^{x}, 1 / f^{(k)}\right)$ is increasing, from the result of (I) and Lemma 8, we get

$$
\lim _{x \rightarrow \infty} F(x) /\left(\frac{N\left(e^{x}, 1 / f^{(k)}\right)^{2}}{N^{\prime}\left(e^{x}, 1 / f^{(k)}\right) e^{x}}\right)=1 .
$$

Now we shall show that $F(x)$ and $G(x)$ satisfy the conditions of Lemma 9 .

(i) From (12) and (13), we get $F^{\prime}(x)=N\left(e^{x}, 1 / f^{(k)}\right), G^{\prime}(x)=A_{0} T\left(e^{x}, f^{(k)}\right)$. Obviously $F^{\prime}(x)$ and $G^{\prime}(x)$ are continuous fuctions.

(ii) Since $T\left(r, f^{(k)}\right)$ is a convex function of $\log r, G^{\prime}(x)>0$. Thus $G(x)$ is an increasing convex function.

(iii) Since $F(x)>0$ and

$$
\frac{d^{2}}{d x^{2}}\left(\frac{1}{F(x)}\right)=\frac{1}{F^{3}(x)}\left\{2 N^{2}\left(e^{x}, \frac{1}{f^{(k)}}\right)-F(x) N^{\prime}\left(e^{x}, \frac{1}{f^{(k)}}\right) e^{x}\right\} .
$$

From (14), if $x$ is sufficiently large, we have

$$
\frac{d^{2}}{d x^{2}}\left(\frac{1}{F(x)}\right)>0
$$

Thus $F(x)$ is a convex function. From the result of (II) and Lemma 9, we obtain $\lim _{r \rightarrow \infty} N\left(r, 1 / f^{(k)}\right) / T\left(r, f^{(k)}\right)=A_{0}$. Similarly, we have $\lim _{r \rightarrow \infty} N\left(r, f^{(k)}\right) /$ $T\left(r, f^{(k)}\right)=B_{0}$. Thus we obtain

$$
K\left(f^{(k)}\right)=\lim _{r \rightarrow \infty} \frac{N\left(r, 1 / f^{(k)}\right)+N\left(r, f^{(k)}\right)}{T\left(r, f^{(k)}\right)}=A_{0}+B_{0}=\frac{2 k(1-\delta(\infty, f))}{1+k-k \delta(\infty, f)} .
$$

The proof of Theorem 1.3 is now complete.

Acknowledgement. The author would like to thank Prof. Seiki Mori for valuable suggestion.

\section{REFERENCES}

[ 1] S. K. Singh AND V. N. Kulkarni, Characteristic function of a meromorphic function and its derivative, Ann. Polon. Math. 28 (1973), 123-133.

[2] Mingliang Fang, A note on a result of Singh and Kulkarni, Internat. J. Math. Sci. Vol. 23 No. 4, (2000), 285-288.

[ 3 ] W. K. Hayman, Meromorphic functions, Clarendon Press, Oxford, 1964. 
[4] M. Furuta AND N. Toda, On exceptional values of a meromorphic function of divergence class, J. Math. Soc. Japan, 25 (4) (1973), 667-679.

[5] WeILING Xiong, Characteristic function of an infinite order meromorphic function and its derivative, Journal of Math. (PRC), Vol. 21 No. 1 (2001), 84-88.

[6] LIANZHONG YANG, Remark on "characteristic function of a meromorphic function and its derivative". Chinese Math. Ann. A 10 (1) (1989), 100-104.

[ 7 ] Lo Yang And Yuefei Wang, Drasin's problem and Mues conjecture, Science in China, A 5 (1992), 455-462.

[8] G. H. Hardy, Orders of infinity, Cambridge University Press, London, 1924.

[9] Pan Cengdong and Yu Xiuyuan, Estmation of stairs, Science and Technology Publishing House of Shan dong, Jinan, (1983), 321-324.

DEPARTMENT OF INFORMATION

AND COMPUTING SCIENCE

GuangXi Institute of TeChNology

545006, Liuzhou GuANGXI

CHINA 